\title{
Efecto del rendimiento del combustible en la decisión de compra de vehículo
}

\author{
Fuel efficiency effect on car buỵing choice \\ Efeito do rendimento do combustível na decisão de compra de \\ veículo
}

\section{Resumen}

El trabajo presenta los resultados de una investigación que se realizó con el propósito de estudiar el efecto del rendimiento del combustible en la adquisición de un vehículo nuevo. La metodología se basó en una encuesta de preferencias declaradas, en el contexto de un experimento de elección de dos alternativas no etiquetadas que se caracterizan por el costo del auto y el rendimiento del combustible. Con los datos acopiados se estimó un modelo Logit Multinomial, que permitió estudiar diferentes escenarios de elección. Se encontró que, en promedio, los individuos están dispuestos a pagar $\$ 1.020 .000$ por mejorar en $1 \mathrm{~km} /$ galón el rendimiento del combustible; este valor puede servir de referencia a la industria automotriz en el esfuerzo por producir vehículos de mayor rendimiento.

Palabras clave: disposición a pagar, encuestas de preferencias declaradas, mercado automotor, rendimiento del combustible.

\section{Abstract}

The results of a research to study the fuel efficiency effect in acquiring a new vehicle are presented. The methodology is based on applying a stated preference survey in the context of a choice experiment with two unlabeled alternatives, which are characterized by the car cost and fuel efficiency. Based on the data collected, a Multinomial Logit model was estimated, which allowed to study different choice scenarios. On average, it was

\footnotetext{
* Esp. Universidad Pedagógica y Tecnológica de Colombia (Tunja - Boyacá, Colombia).julian.morales@uptc.edu.co.

** M.Sc. Universidad Central del Ecuador (Quito, Ecuador).

*** M.Sc. Universidad Pedagógica y Tecnológica de Colombia (Tunja - Boyacá, Colombia). luis.marquez@uptc.edu.co.
} 
found that individuals are willing to pay up to 1.020 .000 Colombian pesos by a new vehicle which improves fuel efficiency at $1 \mathrm{kilometer/gallon}$. This value may be useful as reference for the automotive industry in the effort to produce higher performance vehicles.

Keywords: automotive market, fuel efficiency, stated-preference surveys, willingness to pay.

\section{Resumo}

O trabalho apresenta os resultados de uma pesquisa que se realizou com o propósito de estudar o efeito do rendimento do combustível na aquisição de um veículo novo. A metodologia se baseou em uma sondagem de opinião de preferências declaradas, no contexto de um experimento de escolha de duas alternativas não etiquetadas que se caracterizam pelo custo do auto e o rendimento do combustível. Com os dados coletados estimou-se um modelo Logit Multinomial, que permitiu estudar diferentes cenários de escolha. Encontrou-se que, em média, os indivíduos estão dispostos a pagar $\$ 1.020 .000$ por melhorar em $1 \mathrm{~km} /$ galão o rendimento do combustível; este valor pode servir de referência à indústria automotriz no esforço por produzir veículos de maior rendimento.

Palavras chave: disposição a pagar, mercado automotor, rendimento do combustível, sondagem de opinião de preferências declaradas.

Cómo citar este artículo:

[1] J. E. Morales-Rodríguez, D. J. Ortiz-Durán and L. G. Márquez-Díaz, "Efecto del rendimiento del combustible en la decisión de compra de vehículo", Fac. Ing., vol. 25 (42), pp. 55-66, may.-ago. 2016. 


\section{INTRODUCCIÓN}

La gran dependencia del ser humano hacia los combustibles fósiles ha incrementado de manera inquietante su demanda $\mathrm{y}$ ha desencadenado problemáticas tan graves como la contaminación ambiental y la emisión de gases efecto invernadero, que comprometen las condiciones medioambientales a nivel global en el presente y el futuro. En 2010, por ejemplo, el consumo mundial de energía primaria creció el 5,6\%, que representó el mayor crecimiento porcentual en casi 40 años [1]. Es por esto que el mundo enfrenta actualmente dos importantes problemas: la crisis energética y la contaminación del medioambiente [2]. En consecuencia, se considera pertinente actuar en los sectores de más alto consumo de combustibles fósiles.

El transporte automotor es uno de los sectores que demanda combustible en grandes cantidades [3]; para sortearla, la industria automotriz ha trabajado en los últimos años en procura de estrategias de solución, y es así como algunas empresas han producido vehículos que no emplean combustibles fósiles [4]. En este campo se han realizado investigaciones para estudiar la tenencia y el uso de vehículos de combustibles alternativos, analizando, entre otros aspectos, la disposición a pagar (DAP) por esta clase de vehículos, mediante la aplicación de modelos de elección discreta $[5,6]$.

Otros trabajos han tratado el tema del rendimiento del combustible, analizando la aceptación por los usuarios de vehículos con tecnologías que emplean combustibles más limpios [7,8], intentando priorizar los atributos que se han de tener en cuenta en la compra de vehículos, tales como el costo, el fabricante, la clase de carrocería, las condiciones de seguridad, el espacio interior, la velocidad, el tamaño del vehículo y el color [7]. La mayoría de los estudios revisados se basan en metodologías de modelación discreta $[5,6,8]$, teorías económicas [9] y análisis estadísticos generales [7].

Aunque existen en el ámbito mundial varios trabajos que analizan el efecto del rendimiento del combustible como atributo que incide en la compra del auto, la revisión de literatura solo mostró evidencia de una investigación efectuada en Colombia [10], aunque dicho trabajo está más enfocado al análisis de variables latentes tales como la aceptación de políticas gubernamentales, la preocupación ambiental y la importancia de las características del vehículo. Es por esto que la presente investigación evaluó el efecto del rendimiento del combustible en la decisión de elegir un vehículo nuevo, a partir de la estimación de un modelo de elección discreta, basado en la aplicación de encuestas de preferencias declaradas (PD). El modelo estimado permite analizar el comportamiento de los individuos del entorno colombiano, específicamente de Bogotá, con el fin de verificar si el rendimiento del combustible tiene o no efecto sobre la elección del auto.

Los resultados de esta investigación son de gran importancia porque dan a conocer el comportamiento de los compradores de autos en el país, frente a variaciones en el precio de adquisición y el rendimiento del combustible, lo cual resulta particularmente interesante para la industria automotriz, y tiene efectos en el consumo de combustibles y en los impactos que ese consumo genera.

\section{MARCo teórico}

Las evaluaciones de demanda de transporte se pueden apoyar en modelos de elección discreta, cimentados en la teoría de la maximización de la utilidad aleatoria, los cuales parten de una simplificación de las decisiones individuales para tratar analíticamente su comportamiento [11] y permiten, a través de modelos econométricos, elaborar prognosis probabilísticas de elecciones en situaciones determinadas [12]. Para la calibración de estos modelos es necesario obtener datos mediante la aplicación de encuestas específicas, que pueden ser de preferencias reveladas (PR) o de PD [13].

Aunque se han desarrollado modelos y teorías del comportamiento para tratar de explicar las decisiones que se relacionan con el ámbito económico y psicológico [14], casi siempre la modelación de las decisiones de los individuos sigue una secuencia de elecciones jerarquizadas [15], que puede ser resumida así: definición del problema de elección, generación de alternativas, evaluación de los atributos de las alternativas, elección y ejecución de la alternativa elegida [16].

Teóricamente, en los modelos de elección discreta se representa el comportamiento de un individuo $n$ que se enfrenta a la elección de una única alternativa $i$, entre un conjunto finito $I$ de alternativas disponibles [11]; el 
individuo busca maximizar su utilidad, así que prefiere la alternativa $i$ sobre la $j$ si $U_{n i}>U_{n j}$. El investigador observa solo algunos atributos de decisión $x_{n j}$ y algunos atributos del individuo $s_{n \text {; de manera }}$ que mientras el problema es determinístico para el individuo, no lo es así para el investigador, quien tratará la función de utilidad con un error aleatorio $\varepsilon_{n j}$ de media cero y, por lo tanto,

$$
\mathrm{U}_{\mathrm{nj}}=\mathrm{V}_{\mathrm{nj}}+\varepsilon_{\mathrm{nj}}
$$

Entonces, la utilidad representativa o sistemática $V_{n j}$ es una función que requiere para su calibración el ajuste de los parámetros $\theta$ que acompañan a las variables o atributos incluidos en la función de utilidad determinística [12].

$$
V_{n j}=\sum_{k} \theta_{k} \cdot x_{k n j}
$$

En la mayor parte de los modelos de elección discreta que se emplean en la actualidad, se especifican funciones lineales en los parámetros para representar la utilidad determinística y se considera que los parámetros $\theta$ son constantes para todos los individuos, pero pueden variar entre las alternativas [11]. Los distintos modelos de comportamiento de maximización de la utilidad aleatoria se diferencian principalmente en la especificación que suponen para la densidad de los factores no observados $f\left(\varepsilon_{n}\right)$.

Los principales modelos de elección discreta son:

- Logit multinomial (MNL), con errores IID Gumbel, que no admite correlación entre alternativas ni entre elecciones $\mathrm{y}$ requiere homocedasticidad.

- $\quad$ Probit Binario (BP), cuyos factores no observados distribuyen conjuntamente Normal.

- Modelos de valor extremo generalizados (GEV), que admiten patrones de correlación entre alternativas, tales como el Logit jerárquico $\mathrm{o}$ anidado (HL), en el que las alternativas se estructuran en grupos llamados nidos, de modo que los factores no observados tienen la misma correlación para las alternativas en el interior del nido y no existe correlación con las restantes [12].

- Logit mixto (ML), en donde los factores no observados se dividen en una parte que contiene la posible correlación y heterocedasticidad y otra parte que distribuye Gumbel, con la ventaja de que este modelo se puede aproximar a cualquiera de los otros [11] y, por lo tanto, es completamente general.

La forma general del modelo MNL viene dada por:

$$
P_{n j}=e^{\sum_{k} \theta_{k} \cdot x_{k n j}} / \sum_{i} e^{\sum_{k} \theta_{k} \cdot x_{k n i}}
$$

El proceso de calibración consiste en la estimación de los coeficientes $\theta$, tomando como estimadores aquellos valores que maximicen la verosimilitud de la muestra utilizada [11]. La selección del mejor modelo se basa en el examen y comparación de algunos indicadores tales como la consistencia de signos, la significancia de los estimadores, medidas de bondad de ajuste mediante los índices de razón de verosimilitud $\rho_{\text {y }} \rho^{2}$ [17] y la aplicación del test de razón de verosimilitud para comparar modelos más complejos frente a otros más sencillos que pueden obtenerse mediante restricciones lineales.

Una vez que el mejor modelo es elegido, además de su empleo para realizar pronósticos de elecciones en diferentes escenarios, puede aprovecharse para medir elasticidades con respecto a diferentes variables tales como costos y demás atributos [12]. Básicamente la elasticidad directa, que permite medir la sensibilidad de la demanda ante cambios en el valor de algún atributo, puede ser obtenida así:

$$
E_{j k_{n j}}=-\theta_{k}\left(1-P_{n j}\right) \cdot x_{k n j}
$$

\section{DESARRollo EXPERIMENTAL}

Esta investigación se basó en un experimento de elección, apoyado en encuestas PD, cuyos datos permitieron calibrar modelos discretos, y a partir de la experimentación con el mejor modelo se determinó la DAP de los individuos por mejorar el rendimiento del combustible.

La investigación centró su interés en las tres clases de vehículos particulares más comunes en Colombia: 
automóviles, camperos y camionetas [18]. Para cada clase de vehículo se conformaron dos alternativas no etiquetadas, de naturaleza genérica, que no hacen referencia a ninguna marca comercial, o color en particular. Las dos alternativas se caracterizaron con dos atributos: costo del vehículo y rendimiento del combustible, con variaciones en los niveles según la clase de vehículo considerada.

El diseño se planteó de la forma más sencilla posible para facilitar el proceso de elección por los individuos encuestados. Cada alternativa se representó mediante
2 atributos, y cada atributo con 3 niveles de variación. Para la determinación de los niveles de cada atributo se partió de los valores más representativos en cada tipo de vehículo y se hizo un diseño experimental, basado en el principio de ortogonalidad a partir de las tablas de Koçur et al. [19], obteniendo el plan ortogonal de efectos principales.

La Tabla 1 presenta la relación de atributos y niveles para cada una de las alternativas y clases de vehículos considerados.

\section{TABLA 1}

ATRIBUTOS Y NIVELES PARA CADA UNA DE LAS ALTERNATIVAS

\begin{tabular}{|l|c|c|c|c|c|c|}
\hline \multirow{2}{*}{ Atributos } & \multicolumn{2}{|c|}{ Auto } & \multicolumn{2}{c|}{ Campero } & \multicolumn{2}{c|}{ Camioneta } \\
\cline { 2 - 7 } & Opción 1 & Opción 2 & Opción 1 & Opción 2 & Opción 1 & Opción 2 \\
\hline $\begin{array}{l}\text { Costo del } \\
\text { vehículo } \\
\text { (Millones de } \\
\text { pesos) }\end{array}$ & 25 & 35 & 45 & 55 & 50 & 60 \\
\cline { 2 - 7 } $\begin{array}{l}\text { Rendimiento } \\
\text { combustible }\end{array}$ & 45 & 60 & 70 & 85 & 75 & 90 \\
\cline { 2 - 7 } (km/galón) & 38 & 80 & 100 & 120 & 110 & 130 \\
\cline { 2 - 7 } & 53 & 68 & 18 & 25 & 18 & 25 \\
\hline
\end{tabular}

Con base en los niveles adoptados para cada clase de vehículo, se determinó el diseño experimental, tal como se ve en las Tablas 2, 3 y 4. El diseño inicial de la encuesta se sometió a consideración de un grupo focal y después de los ajustes realizados se aplicó una prueba piloto para verificar la consistencia del experimento mediante una muestra de 31 encuestados, de la cual se obtuvo un total de 27 encuestas útiles, después de haber rechazado las encuestas provenientes de individuos lexicográficos con respecto al costo.
Con los datos provenientes de la encuesta piloto se hizo la estimación de un modelo MNL sencillo y se pudo comprobar la consistencia de los signos de los parámetros estimados, así como su significancia estadística. Comprobada la consistencia del modelo y del experimento se procedió a aplicar la encuesta definitiva en la ciudad de Bogotá, tomando una muestra total de 100 individuos no lexicográficos, que en conjunto aportaron 900 observaciones para la calibración de modelos, en virtud de las nueve situaciones declaradas por cada uno de ellos. 


\section{TABLA 2}

DiSEÑO EXPERIMENTAL PARA EL TIPO DE VEHÍCULO AUTOMÓVIL

\begin{tabular}{|c|c|c|c|}
\hline \multicolumn{2}{|c|}{ Automóvil 1 } & \multicolumn{2}{c|}{ Automóvil 2 } \\
\hline $\begin{array}{c}\text { Costo vehículo } \\
\text { (millones) }\end{array}$ & Rendimiento (km/galón) & $\begin{array}{c}\text { Costo vehículo } \\
\text { (millones) }\end{array}$ & $\begin{array}{c}\text { Rendimiento (km/ } \\
\text { galón) }\end{array}$ \\
\hline 25 & 28 & 35 & 35 \\
\hline 25 & 38 & 35 & 48 \\
\hline 25 & 53 & 35 & 66 \\
\hline 45 & 28 & 60 & 35 \\
\hline 45 & 38 & 60 & 48 \\
\hline 45 & 53 & 60 & 66 \\
\hline 60 & 28 & 80 & 35 \\
\hline 60 & 38 & 80 & 48 \\
\hline 60 & 53 & 80 & 66 \\
\hline
\end{tabular}

TABLA 3

DISEÑO EXPERIMENTAL PARA EL TIPO DE VEHÍ́CULO CAMPERO

\begin{tabular}{|c|c|c|c|}
\hline \multicolumn{2}{|c|}{ Campero 1 } & \multicolumn{2}{c|}{ Campero 2 } \\
\hline $\begin{array}{c}\text { Costo vehículo } \\
\text { (millones) }\end{array}$ & Rendimiento (km/galón) & $\begin{array}{c}\text { Costo vehículo } \\
\text { (millones) }\end{array}$ & $\begin{array}{c}\text { Rendimiento (km/ } \\
\text { galón) }\end{array}$ \\
\hline 45 & 18 & 55 & 25 \\
\hline 45 & 28 & 55 & 38 \\
\hline 45 & 38 & 55 & 51 \\
\hline 70 & 18 & 85 & 25 \\
\hline 70 & 28 & 85 & 38 \\
\hline 70 & 38 & 85 & 51 \\
\hline 100 & 18 & 120 & 25 \\
\hline 100 & 28 & 120 & 38 \\
\hline 100 & 38 & 120 & 51 \\
\hline
\end{tabular}




\section{TABLA 4}

DiSEÑO EXPERIMENTAL PARA EL TIPO DE VEHÍCULO CAMIONETA

\begin{tabular}{|c|c|c|c|}
\hline \multicolumn{2}{|c|}{ Camioneta 1 } & \multicolumn{2}{c|}{ Camioneta 2 } \\
\hline $\begin{array}{c}\text { Costo vehículo } \\
\text { (millones) }\end{array}$ & Rendimiento (km/galón) & $\begin{array}{c}\text { Costo vehículo } \\
\text { (millones) }\end{array}$ & $\begin{array}{c}\text { Rendimiento (km/ } \\
\text { galón) }\end{array}$ \\
\hline 50 & 18 & 60 & 25 \\
\hline 50 & 23 & 60 & 33 \\
\hline 50 & 33 & 60 & 46 \\
\hline 75 & 18 & 90 & 25 \\
\hline 75 & 23 & 90 & 33 \\
\hline 75 & 33 & 90 & 46 \\
\hline 110 & 18 & 130 & 25 \\
\hline 110 & 23 & 130 & 33 \\
\hline 110 & 33 & 130 & 46 \\
\hline
\end{tabular}

También se tomó información para caracterizar a los individuos y sus vehículos mediante atributos como género, ingreso del encuestado, clase de vehículo, caja de cambios, antigüedad del vehículo, asiduidad y ámbito de uso del vehículo. La distribución muestral de estas variables se puede ver en la Fig. 1. La distribución muestral de las variables se considera consistente con la distribución de la población usuaria de auto en Bogotá [20].

Con los datos acopiados, mediante la aplicación del software BIOGEME [21], se hizo la estimación de modelos discretos. Las variables específicas consideradas para la estimación de modelos son presentadas en la Tabla 5 .

TABLA 5

DEFINICIÓN DE VARIABLES PARA LA ESTIMACIÓN DE MODELOS

\begin{tabular}{|l|l|l|}
\hline \multicolumn{1}{|c|}{ Variable } & \multicolumn{2}{c|}{ Valores } \\
\hline Género & 0: Femenino & 1: Masculino \\
\hline Ingreso & 0: Menor o igual a 2 millones & 1: Mayor a 2 millones \\
\hline Antigüedad del vehículo & 0: Menor a 5 años & 1: Mayor o igual a 5 años \\
\hline Caja de cambios & 0: Manual & 1: Automática \\
\hline Ámbito de uso del vehículo & 0: Combinado (urbano e interurbano) & 1: Otro \\
\hline Asiduidad de uso vehículo & 0: Todos los días & 1: Otro \\
\hline
\end{tabular}




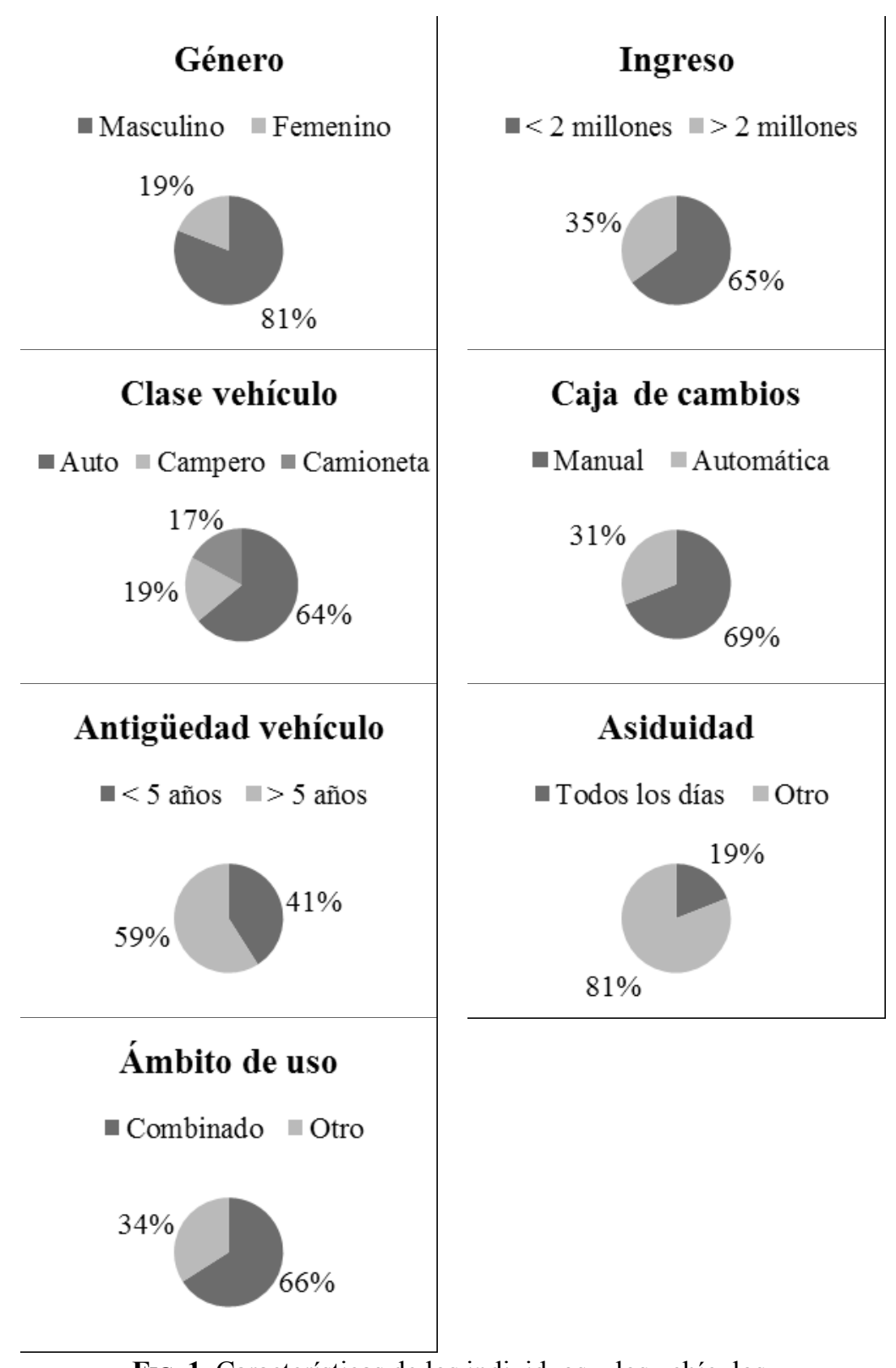

Fig. 1. Características de los individuos y los vehículos.

\section{REsultados}

Los mejores modelos obtenidos se muestran en la Tabla 6, todos ellos con signos consistentes con la teoría microeconómica subyacente y coeficientes altamente significativos. Aunque fueron calibrados modelos de tipo ML, ninguna de las especificaciones probadas permitió obtener modelos de calidad, debido a que los componentes aleatorios establecidos en las funciones de utilidad no fueron significativos. También fueron probados modelos con variaciones sistemáticas en los gustos de los usuarios, pero las interacciones propuestas no resultaron significativas.

La Tabla 6 está conformada por dos partes. La parte superior presenta los coeficientes estimados, junto con el estadístico $t$ entre paréntesis, que permite verificar la significancia de cada coeficiente estimado. La segunda parte muestra las medidas de bondad de ajuste de los modelos y el ranking establecido según la calidad de cada uno de ellos. 


\section{TABLA 6}

Modelos estimados

\begin{tabular}{|c|c|c|c|c|c|}
\hline \multicolumn{2}{|c|}{ Coeficientes estimados } & MNL-1 & MNL-2 & BP-1 & BP-2 \\
\hline Costo del vehículo $(1,2)$ & $\theta_{c}$ & $\begin{array}{c}-0,0716 \\
(-5,54)\end{array}$ & $\begin{array}{c}-0,0848 \\
(-6,19)\end{array}$ & $\begin{array}{c}-0,0443 \\
(-5,60)\end{array}$ & $\begin{array}{c}-0,0524 \\
(-6,28)\end{array}$ \\
\hline Rendimiento del combustible $(1,2)$ & $\theta_{r}$ & $\begin{array}{l}0,104 \\
(5,32)\end{array}$ & $\begin{array}{c}0,0831 \\
(4,02)\end{array}$ & $\begin{array}{c}0,0640 \\
(5,37)\end{array}$ & $\begin{array}{l}0,0510 \\
(4,03)\end{array}$ \\
\hline Ámbito de uso vehículo (1) & $\theta_{a}$ & & $\begin{array}{l}0,341 \\
(2,12)\end{array}$ & & $\begin{array}{l}0,210 \\
(2,11)\end{array}$ \\
\hline Asiduidad de uso vehículo (1) & $\theta_{f}$ & & $\begin{array}{l}-0,746 \\
(-4,45)\end{array}$ & & $\begin{array}{l}-0,464 \\
(-4,49)\end{array}$ \\
\hline Caja de cambios (1) & $\theta_{v}$ & $\begin{array}{l}-0,345 \\
(-2,35)\end{array}$ & & $\begin{array}{l}-0,213 \\
(-2,35)\end{array}$ & \\
\hline \multicolumn{6}{|l|}{ Medidas de bondad de ajuste } \\
\hline Log-verosimilitud inicial & $l(0)$ & $-623,832$ & $-623,832$ & $-623,832$ & $-623,832$ \\
\hline Log-verosimilitud final & $l(\theta)$ & $-604,610$ & $-595,548$ & $-604,684$ & $-595,598$ \\
\hline$\rho^{2}$ ajustado & $\rho^{2}$ & 0,026 & 0,039 & 0,026 & 0,039 \\
\hline $\mathrm{N} .^{\circ}$ de parámetros & $k$ & 3 & 4 & 3 & 4 \\
\hline $\mathrm{N} .^{\circ}$ de observaciones & $n$ & 900 & 900 & 900 & 900 \\
\hline \multicolumn{2}{|l|}{ Ranking } & 3 & 1 & 4 & 2 \\
\hline
\end{tabular}

Dado que la encuesta PD utilizó un experimento no etiquetado, la especificación de la utilidad sistemática de los modelos probados no consideró la estimación de constantes modales, ya que sería teóricamente incorrecto. El signo negativo del coeficiente del costo del vehículo es correcto, dado que, en la medida que el costo del vehículo sea mayor, la utilidad individual disminuye. El signo positivo del coeficiente del rendimiento del combustible también es correcto, pues un vehículo con mayor rendimiento le representa mayor utilidad al usuario.

Los atributos individuales asociados con el ámbito de uso del vehículo, la asiduidad de uso del vehículo y la caja de cambios fueron especificados únicamente en la alternativa 1, que, como se puede ver en las Tablas 2, 3 y 4, corresponde a la alternativa de mayor costo. Es evidente que estos atributos son determinantes al momento de comprar un vehículo nuevo.
La relación existente entre el coeficiente del rendimiento de combustible y el coeficiente del costo en el mejor modelo dio como resultado que la DAP de los individuos por mejorar el rendimiento de combustible en $1 \mathrm{~km} /$ galón es de $\$ 1.020 .000$. Este resultado es consistente con el presentado por Espey [22], quien sugirió un valor de 560 US\$ por mejorar el rendimiento en 1 milla/galón, tomando como referencia un precio de 1.5 US\$/galón. No obstante, el resultado difiere un poco del rango de valores mostrado en [9].

La elasticidad entre el rendimiento de combustible y la demanda por un auto nuevo, calculada por enumeración muestral, dio como resultado un valor de $-2,5922$, el cual se considera razonable, aunque sutilmente alto, si se compara con el rango de valores reportados en referentes internacionales [23], que oscilan entre $-1,515$, para vehículos de gran rendimiento, y -2,297, para vehículos de bajo rendimiento. 
Se determinó la variación de la elasticidad en función del rendimiento de combustible del vehículo tomado como referencia, encontrando que la mayor elasticidad, en términos absolutos, se tendría para aquellos vehículos con rendimientos menores, lo cual es consistente con la teoría subyacente al modelo y con la literatura internacional [23], aunque el orden de magnitud es diferente, tal como se ve en la Fig. 2.

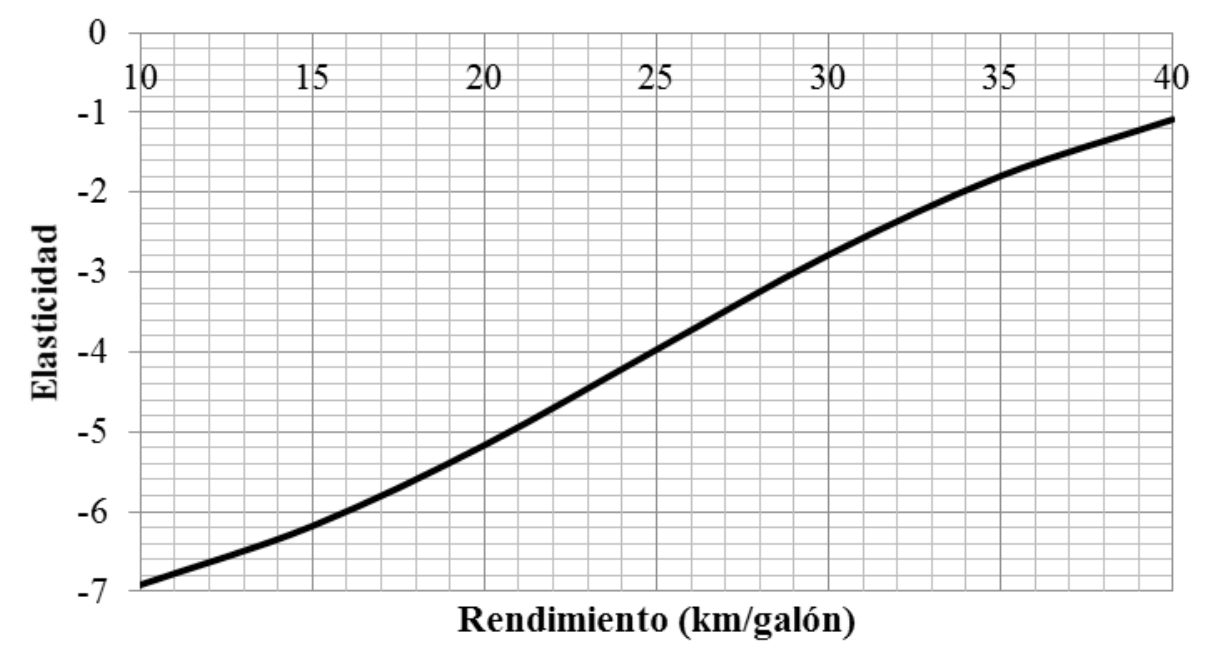

Fig. 2. Elasticidad en función del rendimiento del vehículo de referencia.

También se pudo establecer que, a mayor costo de adquisición del vehículo, los compradores se hacen más sensibles al efecto del rendimiento, aumentando considerablemente la elasticidad, en términos absolutos, como se ve en la Fig. 3.

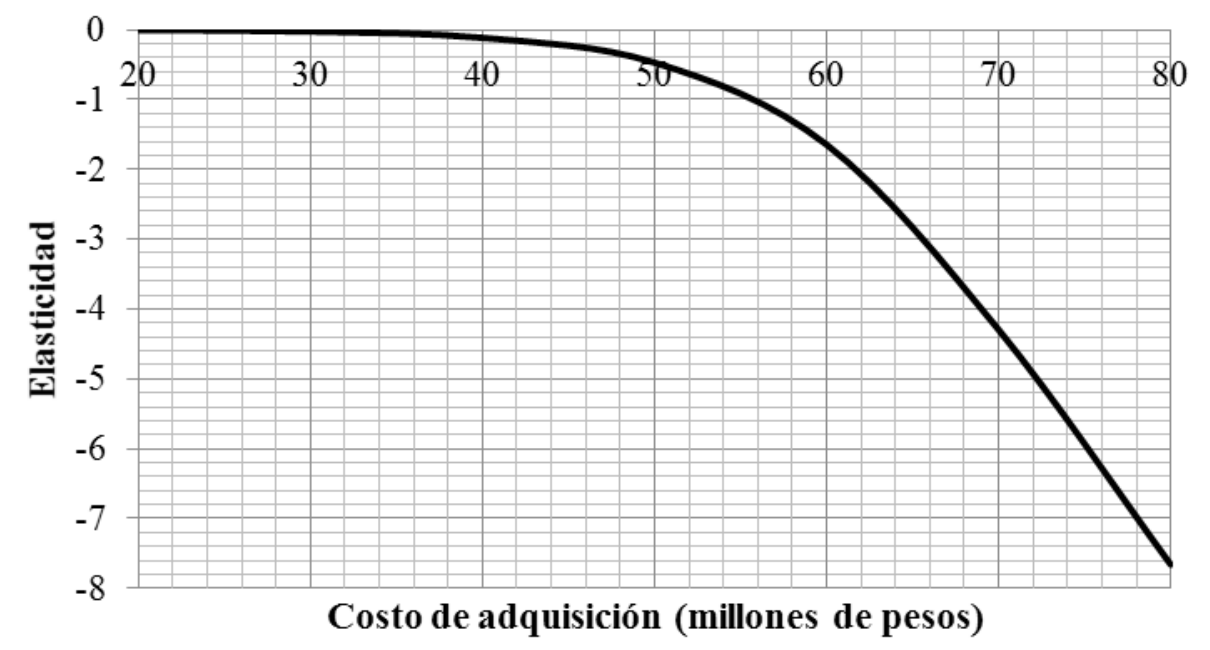

Fig. 3. Variación de la elasticidad en función del costo de adquisición.

\section{Conclusiones}

El modelo Logit multinomial elegido permite concluir que el proceso de elección para la compra de un vehículo nuevo está determinado por el costo del vehículo, el rendimiento del combustible, el ámbito de uso del vehículo, la asiduidad del uso del vehículo y el tipo de caja de cambios. Todos estos atributos son determinantes y estadísticamente significativos al $1 \%$. 
Los parámetros del modelo permitieron determinar que la DAP de los individuos por mejorar el rendimiento de combustible en $1 \mathrm{~km} /$ galón es de $\$ 1.020 .000$. Este resultado se considera de mucha importancia tanto para los productores de combustibles como para la industria automotriz, ya que sienta las bases para evaluar estrategias que mejoren el rendimiento de los combustibles a cambio de un mayor precio de venta de los vehículos automotores nuevos.

Con base en la evaluación de los valores promedio de las variables en el modelo se obtuvo una elasticidad entre el rendimiento de combustible y la demanda por un auto nuevo de -2,5922. Se pudo comprobar, además, que la mayor elasticidad, en términos absolutos, se tiene para aquellos vehículos con rendimientos menores, y que, en la medida que aumenta el costo de adquisición del vehículo, la elasticidad del rendimiento del combustible aumenta. El valor de elasticidad que se obtuvo y el comportamiento del modelo corresponden con los resultados de otros estudios y, por lo tanto, se consideran aceptables.

Aunque el modelo estimado tiene un nivel de confianza superior al $98 \%$, no se puede afirmar que los resultados obtenidos sean plenamente concluyentes, así que se recomienda aplicar el estudio en otros contextos y examinar con mayor detalle el efecto que tendría sobre la compra de vehículos nuevos el mejoramiento del rendimiento de combustible.

\section{REFERENCIAS}

[1] C. S Jones and S. P. Mayfield, "Algae biofuels: versatility for the future of bioenergy", Current Opinion in Biotechnology, vol. 23 (3), pp. 346351, Jun. 2012. DOI: http://dx.doi.org/10.1016/j. copbio.2011.10.013.

[2] V. K. Gupta and M. G. Tuohy, Biofuel technologies: recent developments. Berlin: Springer Berlin Heidelberg, 2013.

[3] B. Lin and C. Xie, "Estimation on oil demand and oil saving potential of China's road transport sector", Energy Policy, vol. 61, pp. 472-482, Oct. 2013. DOI: http://dx.doi.org/10.1016/j. enpol.2013.06.017.

[4] B. Rajesh Kumar and S. Saravanan, "Use of higher alcohol biofuels in diesel engines: A review", Renewable and Sustainable Energy Reviews, vol. 60, pp. 84-115, Jul. 2016. DOI: http://dx.doi.org/10.1016/j.rser.2016.01.085.
[5] J. Ahn, G. Jeong and Y. Kim, "A forecast of household ownership and use of alternative fuel vehicles: A multiple discrete-continuous choice approach", Energy Economics, vol. 30 (5), pp. 2091-2104, Sep. 2008. DOI: http://dx.doi. org/10.1016/j.eneco.2007.10.003.

[6] C. Erdem, I. Sentürk and T. Simsek, "Identifying the factors affecting the willingness to pay for fuel-efficient vehicles in Turkey: a case of hybrids", Energy Policy, vol. 38 (6), pp. 3038-3043, Jun. 2010. DOI: http://dx.doi. org/10.1016/j.enpol.2010.01.043.

[7] T. Sakaguchi, "Influence of diffusion of fuelefficient motor vehicles on gasoline demand for individual user owned passenger cars", Energy Policy, vol. 28 (12), pp. 895-903, Oct. 2000. DOI: http://dx.doi.org/10.1016/S03014215(00)00071-9.

[8] D. Brownstone, D. S. Bunch and K. Train, "Joint mixed logit models of stated and revealed preferences for alternative-fuel vehicles", Transportation Research Part B: Methodological, vol. 34 (5), pp. 315-338. Jun. 2000. DOI: http://dx.doi.org/10.1016/S01912615(99)00031-4.

[9] T. S. Turrentine and K. S. Kurani, "Car buyers and fuel economy?", Energy Policy, vol. 35 (2), pp. 1213-1223. Feb. 2007. DOI: http://dx.doi. org/10.1016/j.enpol.2006.03.005.

[10] J. J. Soto, V. Cantillo and J. Arellana, “ Modelación híbrida para la elección de vehículos con energías alternativas", Interciencia, vol. 39 (9), pp. 666-672. Sep. 2014.

[11] K. Train, Discrete choice methods with simulation. United Kingdom: Cambridge University Press, 2003. DOI: http://dx.doi. org/10.1017/CBO9780511753930.

[12] A. Orro, "Modelos de elección discreta en transportes con coeficientes aleatorios". Tesis de doctorado, Facultad de Ingeniería Civil, Universidad de la Coruña, Coruña, España, 2005.

[13] J. J. Louviere, R. J. Meyer, D. S. Bunch et al., "Combining Sources of Preference Data for Modeling Complex Decision Processes", Marketing Letters, vol. 10 (3), pp. 205-217, Aug. 1999. DOI: http://dx.doi. org/10.1023/A:1008050215270.

[14] D. Kahneman, "Maps of bounded rationality, prize lecture". In: T. Frängsmyr (ed), Les Prix Nobel: The Nobel Prizes 2002. Stockholm: Nobel Foundation, 2003. 
[15] J. Bates, "History of demand modelling". In: D. A. Hensher and K. J. Button (ed), Handbook of transport modelling, pp. 11-33. Amsterdam: Pergamon, 2000.

[16] M. Ben-Akiva and S. R. Lerman, Discrete choice analysis, theory and application to travel demand. Cambridge, Massachusetts: The MIT Press, 1985.

[17] J. D. Ortúzar and L. G. Willumsen, Modelling transport. Chichester: John Wiley \& Sons, 2011.

[18] J. Téllez, M. Hernández, M. C. Llanes et al., Situación automotriz Colombia. Bogotá: BBVA Research, 2012.

[19] G. Koçur, T. Adler, W. Hyman and E. Audet, Guide to Forecasting Travel Demand with Direct Utility Measurement. Washington D.C.: USA Department of Transportation, 1982.
[20] L. Márquez, L. A. Gallo and C. A. Chacón, "Influence of Parking Costs on the Use of Cars in Bogota", Ing. Univ. Bogotá, vol. 15 (1), pp. 105-124, Jan. - Jun. 2011.

[21] M. Bierlaire, "A free package for the estimation of discrete choice models", Proceedings of the 3rd Swiss Transportation Research Conference, Ascona, Switzerland, 2003.

[22] M. Espey, "Do Consumers Value Fuel Economy?", Regulation, Winter 2005-2006, pp. 8-10, 2006.

[23] P. S. McCarthy and R. S. Tay, "New vehicle consumption and fuel efficiency: A nested Logit approach", Transportation Research Part E: Logistics and Transportation Review, vol. 34 (1), pp. 39-51, Mar. 1998. DOI: http://dx.doi. org/10.1016/S1366-5545(97)00042-2. 